\title{
Role of Stat3 Signaling in Control of EMT of Tubular Epithelial Cells During Renal Fibrosis
}

\author{
Jingsong Liu Ying Zhong $^{\mathrm{a}}$ Guoyong Liu ${ }^{\mathrm{b}} \quad$ Xiaobai Zhang ${ }^{\mathrm{a}}$ Bofei Xiao ${ }^{\mathrm{a}}$ \\ Shang Huanga Hong Liuc Liyu Hec \\ aDepartment of Nephrology, Hospital affiliated to Hunan Academy of Chinese Medicine, Chinese \\ Medicine and Western Medicine Hospital affiliated to Hunan University of Chinese medicine, \\ Changsha, 'bepartment of Nephrology, the First Affiliated Hospital of Changde Vocational Technical \\ College, Changde, 'Department of Nephrology, The Second Xiangya Hospital of Central South \\ University, Key Lab of Kidney Disease and Blood Purification in Hunan, Changsha, China
}

\section{Key Words}

Stat3 • Renal fibrosis $•$ TGF $\beta 1 \cdot$ Epithelial-to-mesenchymal transition

\begin{abstract}
Background/Aims: Transforming growth factor $\beta 1$ (TGF $\beta 1$ ) plays a critical role in the epithelial-to-mesenchymal transition (EMT) of renal tubular epithelial cells (TECs) during renal injury, a major cause of acute renal failure, renal fibrosis and obstructive nephropathy. However, the underlying molecular mechanisms remain ill-defined. Here, we addressed this question. Methods: Expression of TGF $\beta 1$, Snail, and phosphorylated Stat3 was examined by immunohistochemistry in the kidney after induction of unilateral ureteral obstruction (UUO) in mice. In vitro, primary TECs were purified by flow cytometry, and then challenged with TGF $\beta 1$ with/without presence of specific inhibitors for phosphorylation of SMAD3 or Stat3. Protein levels were determined by Western blotting. Results: We detected significant increases in Snail and phosphorylated Stat3, an activated form for Stat3, in the kidney after induction of UUO in mice. In vitro, TGFß1-challenged primary TECs upregulated Snail, in a SMAD3/Stat3 dependent manner. Conclusion: Our study sheds light on the mechanism underlying the EMT of TECs after renal injury, and suggests Stat3 signaling as a promising innovative therapeutic target for prevention of renal fibrosis.

(C) 2017 The Author(s)

Published by S. Karger AG, Basel
\end{abstract}

\section{Introduction}

The progression of obstructive nephropathy towards end-stage renal failure is associated with epithelial-to-mesenchymal transition (EMT) of renal tubular epithelial cells (TECs), and appearance of alpha smooth muscle actin ( $\alpha$-SMA)-positive myofibroblasts in the damaged region of the kidney [1-5]. Both in vivo and in vitro models suggest that the acquisition of the $\alpha$-SMA-positive phenotype by TECs may be secondary to EMT [6].

Liyu $\mathrm{He}$

KARGER
Department of Nephrology, The Second Xiangya Hospital of Central South University, Key Lab of Kidney Disease and Blood Purification in Hunan, 139 Renminzong Road, Changsha, (China); Tel. +8673285292064; E-Mail zying671@yeah.net 
The EMT progress is mediated by coordination of key transcription factors, including Snail, zinc-finger E-box-binding (ZEB) and basic helix-loop-helix transcription factors. Among these factors, Snail, as an important suppression of E-cadherin, plays a critical role [7-9]. Transforming growth factor $\beta 1$ (TGF $\beta 1$ ) is also a key player in the coordination of the process of EMT of renal TECs during renal injury. Binding of TGF $\beta 1$ onto the TGF $\beta$ receptors on the surface of TECs leads to phosphorylation of a downstream factor, SMAD3 [10]. Phosphorylation of SMAD3 has been regarded as a key progress for the TECs to undergo EMT, since it forms complexes with Smad4 and then translocate into the nucleus and conjunct with other transcription factors to activate target genes [10]. However, the exact molecular signaling that regulates activation of EMT-associated genes by phosphorylation of SMAD3 has not been elucidated. Very recently, activated TGF $\beta 1 /$ SMAD3 signaling has been shown to induce phosphorylation and activation of signal transducer and activator of transcription 3 (Stat3) to promote transcription of Snail in tumors [11]. However, whether this mechanism also functions during EMT of renal TECs is unknown. Here, we addressed this question.

We examined expression of TGF $\beta 1$, Snail, and phosphorylated Stat3 by immunohistochemistry in the kidney after induction of unilateral ureteral obstruction (UUO) in mice. In vitro, primary TECs were purified by flow cytometry, and then challenged with TGF $\beta 1$ with/ without presence of specific inhibitors for phosphorylation of SMAD3 or Stat3. Protein levels were determined by Western blotting.

\section{Materials and Methods}

\section{Protocol approval}

All the experimental methods have been approved by the research committee at Hunan University of Chinese medicine. All animal experiments were approved by the Institutional Animal Care and Use Committee at Hunan University of Chinese medicine (Animal Welfare Assurance). All the experiments and methods were carried out in "accordance" with the approved guidelines. Surgeries were performed in accordance with the Principles of Laboratory Care, supervised by a qualified veterinarian.

\section{The UUO model}

Ten week-old male C57/BL6 mice (SLAC Laboratory Animal Co. Ltd, Shanghai, China) were used in the current study. Ten mice received UUO and 10 mice were sham treated as controls. For UUO, mice received left ureteral ligation with 6-G silk suture, as has been previously described [12]. After surgery, mice were sacrificed at 3 or 14 days after surgery.

\section{Cell isolation, culture and treatment}

Mouse kidney was digested into single cells for flow cytometry. Briefly, de-capsulated mouse kidneys were chopped into small pieces and digested with $60 \mathrm{mg} / \mathrm{dl}$ collagenase (Sigma-Aldrich, San Jose, CA, USA) for 30 minutes in a $37^{\circ} \mathrm{C}$ shaker at $250 \mathrm{rpm}$, after which the digests passed a $40 \mathrm{~nm}$ filter to collect single cell preparation. The single cell faction was then incubated with PEcy7-conjugated anti-E-cadherin (E-cad, Becton-Dickinson Biosciences, San Jose, CA, USA) for sorting of renal TECs by flow cytometry. The sorted cells were either immediately analyzed by RT-qPCR or Western blotting, or cultured in RPMI1640 medium (Invitrogen, Carlsbad, CA, USA) suppled with 10\% fetal bovine serum (FBS, Sigma-Aldrich), penicillin $(100 \mu \mathrm{g} / \mathrm{ml})$ and streptomycin $(250 \mathrm{ng} / \mathrm{ml})$ in a humidified chamber with $5 \% \mathrm{CO}_{2}$ at $37^{\circ} \mathrm{C}$ for other assays. After overnight culture, TECs were treated with/without $10 \mathrm{ng} / \mathrm{ml}$ TGF $\beta 1$ (with/without SMAD3/Stat3 inhibitors) and harvested after $0,1,2,4,8,16$ and 32 hours, respectively. SMAD3 inhibitor SIS3 (R\&D Sytems, Carpinteria, CA, USA; used at $5 \mu \mathrm{mol} / \mathrm{l}$ ) or Stat3 inhibitor NSC74859 (R\&D Sytems; used at $5 \mu \mathrm{mol} / \mathrm{l}$ ) or control DMSO solution was added together with the TGF $\beta 1$.

Quantitative RT-PCR (RT-qPCR)

Total RNA were extracted using miRNeasy mini kit (Qiagen, Hilden, Germany). Complementary DNA (cDNA) was prepared from $2 \mu$ g of total RNA using High-Capacity cDNA Reverse Transcription Kit (Applied Biosystems, Foster City, CA, USA). RT-qPCR was performed in duplicate using QuantiTect SYBR 


\section{Cellular Physiology Cell Physiol Biochem 2017;42:2552-2558 \\ \begin{tabular}{l|l|l} 
and Biochemistry Published online: August 23, 2017 & $\begin{array}{l}\text { (c) } 2017 \text { The Author(s). Published by S. Karger AG, Basel } \\
\text { www.karger.com/cpb }\end{array}$
\end{tabular}}

Liu et al.: Stat3 Signaling Regulates RF-Associated EMT of Tubular Ecs

Green PCR Kit (Qiagen). Primers for mouse TGF $\beta 1$ (QT00145250), E-cadherin (QT00121163) and $\alpha$-tubulin

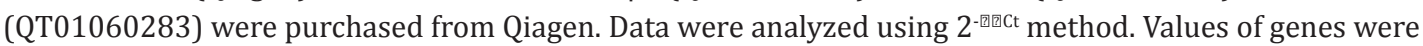
determined by sequential normalization to $\alpha$-tubulin and experimental controls.

Western blot

The protein was extracted using RIPA lysis buffer (Sigma-Aldrich) suppled with proteinase inhibitor (Roche, Nutley, NJ, USA). Primary antibodies were rabbit anti-Snail, anti-SMAD3, anti-phosphorylated SMAD3 (pSMAD3), anti-Stat3, anti-phosphorylated Stat3 (pStat3) (Cell signaling, San Jose, CA, USA). Secondary antibody is HRP-conjugated anti-rabbit (Jackson ImmunoResearch Labs, West Grove, PA, USA). The protein levels were determined after sequential normalization to $\alpha$-tubulin and experimental controls. Densitometry of Western blots was quantified with NIH ImageJ software (Bethesda, MD, USA).

\section{Immunohistochemistry and histology for renal fibrosis}

Kidneys were removed, formalin fixed, embedded in paraffin, sectioned at $5 \mu \mathrm{m}$ thickness, and stained with Masson trichrome using routine procedures. Evaluation of renal fibrosis used 20 randomly selected fields per section from 3 sections that are $50 \mu \mathrm{m}$ to each other. The degree of renal fibrosis was scored as the ratio of the positive stain area (blue) to that of the whole area. For immunohistochemistry, primary antibodies are: rabbit anti-TGFß1 (R\&D Sytems), rabbit anti-Snail (Cell signaling), and rabbit anti-pStat3 (Cell signaling). Secondary antibody is HRP-conjugated rabbit-specific (Jackson ImmunoResearch Labs, West Grove, PA, USA). For ABC staining, incubation with HRP-conjugated secondary antibodies was followed by DAB development (Dako).

\section{Statistical analysis}

GraphPad Prism 6.0 (GraphPad Software, Inc. La Jolla, CA, USA) was used for statistical analyses. All values were depicted as mean \pm standard deviation and were considered significant if $\mathrm{p}<0.05$. All data were statistically analyzed using one-way ANOVA with a Bonferroni correction, followed by Fisher's Exact Test for comparison of two groups.

\section{Results}

\section{Levels of Snail and pStat3 increase in kidney after UUO}

Very recently, activated TGF 1 /SMAD3 signaling has been shown to induce phosphorylation and activation of Stat3 to promote transcription of Snail in tumors [11]. However, whether this mechanism also functions during EMT of renal TECs is unknown. Here, we addressed this question. We used UUO model to mimic the pathological changes during renal injury. We did UUO in 10 mice and sham operated another 10 mice as controls. The mice were sacrificed at 14 days after UUO for renal histology using Masson trichrome staining to analyze and score for the degree of renal fibrosis regions, and for immunohistochemistry for Stat3 activation (Fig. 1A). We found that UUO induced significant increases in kidney fibrosis in 14 days, shown by representative Masson trichrome images (Fig. 1B), and by quantification (Fig. 1C). Moreover, signals for TGF $\beta 1$ (Fig. 1D), Snail (Fig. 1E) and phosphorylated Stat3 (pStat3, an activated form for Stat3; Fig. 1F) all increased after UUO, compared to kidneys from control sham-treated mice, shown by representative images. These data suggest a possible association of Stat 3 signaling and TGF $\beta 1$-induced Snail activation.

\section{TGFB1 activates Snail in renal TECs}

In order to figure out whether TGF $\beta 1$ may trigger EMT of renal TECs through Stat3 signaling cascades, we performed in vitro challenge of TGF $\beta 1$ together with pathway specific inhibitors on primary TECs. First, we digested the mouse kidney and sorted renal TECs by FACS based on their expression of E-cadherin (E-cad) (Fig. 2A), and confirmed the purity by RT-qPCR for E-cad (Fig. 2B). The purified renal TECs were put in culture (Fig. 2C). Next, we treated cultured primary TECs with $10 \mathrm{ng} / \mathrm{ml} \mathrm{TGF} \beta 1$ and harvested the cells at $0,1,2,4$, 8, 16 and 32 hours after TGF $\beta 1$ administration for RT-qPCR and Western blotting for Snail expression. We found that both Snail mRNA (Fig. 2D) and protein (Fig. 2E) were induced 


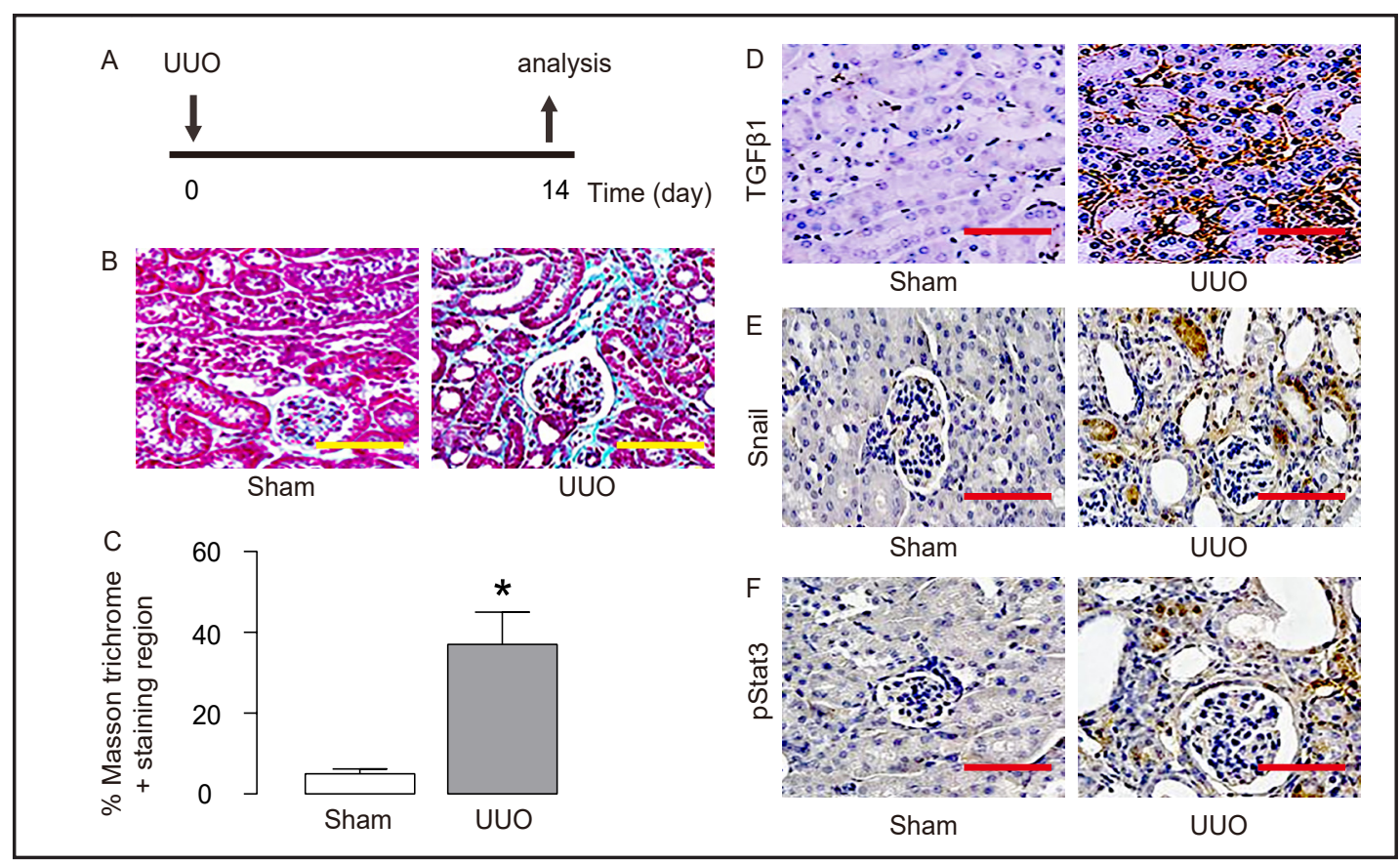

Fig. 1. Levels of Snail and pStat3 increase in kidney after UUO(A) Experimental design: We did UUO in 10 mice and sham operated another 10 mice as controls. The mice were sacrificed at 14 days after UUO for renal histology using Masson trichrome staining to analyze and score for the degree of renal fibrosis regions, and for immunohistochemistry for Stat3 activation. (B-C) Masson trichrome staining for determining the degree of renal fibrosis, shown by representative images (B), and by quantification (C). (D-F) Representative images for TGF $\beta 1$ (D), Snail (1E) and phosphorylated Stat3 (pStat3, F) in kidney from UUO, or control shamtreated mice, 14 days after surgery. $\mathrm{N}=10 .{ }^{*} \mathrm{p}<0.05$.

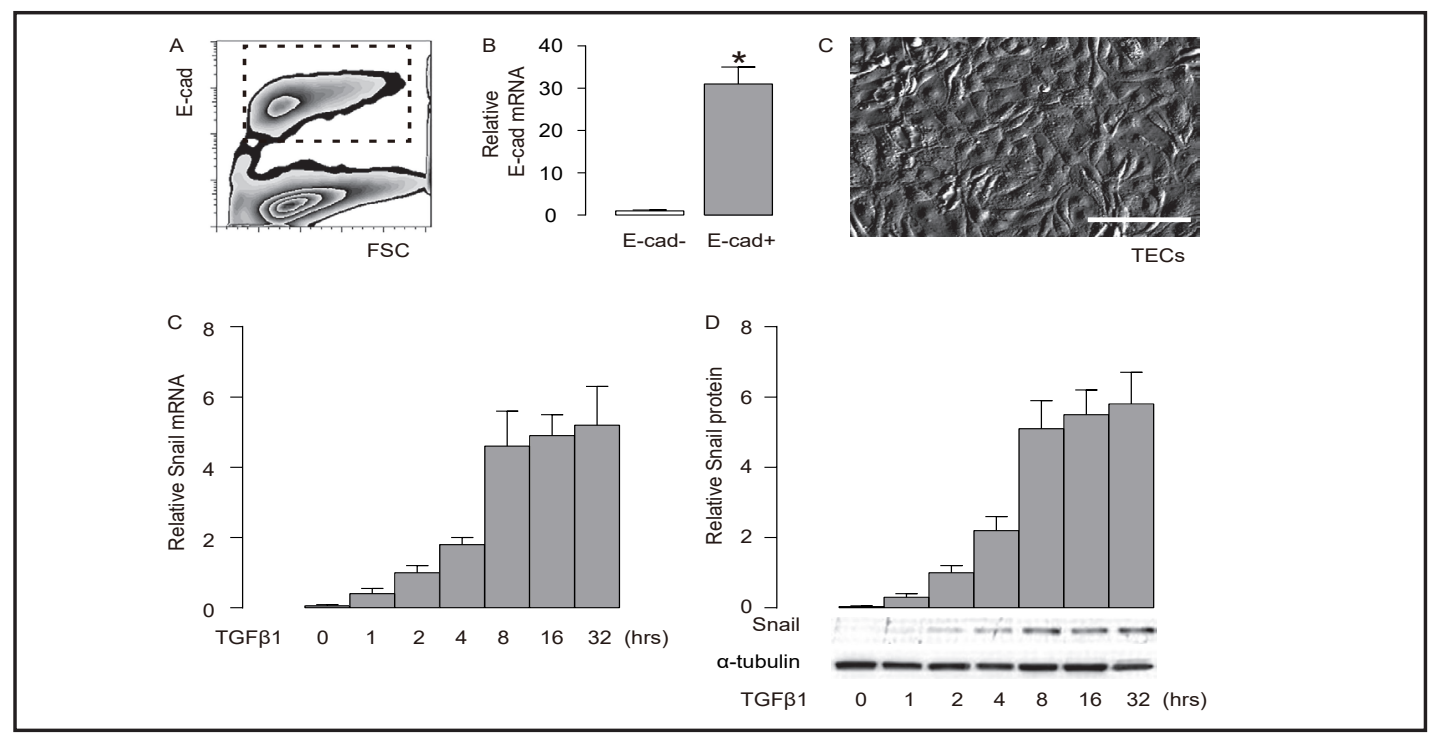

Fig. 2. TGF $\beta 1$ activates Snail in renal TECs(A) Mouse kidney digests were sorted for renal TECs by FACS based on their expression of E-cadherin (E-cad), shown by a representative flow chart. (B) RT-qPCR for E-cad in sorted E-cad- population and E-cad+ population. (C) The purified renal TECs were put in culture. (C-D) We treated cultured primary TECs with $10 \mathrm{ng} / \mathrm{ml}$ TGF $\beta 1$ and harvested the cells at 0, 1, 2, 4, 8, 16 and 32 hours after TGF $\beta 1$ administration for RT-qPCR (C) and Western blotting (D) for Snail expression. N=5. Scale bar is $10 \mu \mathrm{m}$. 


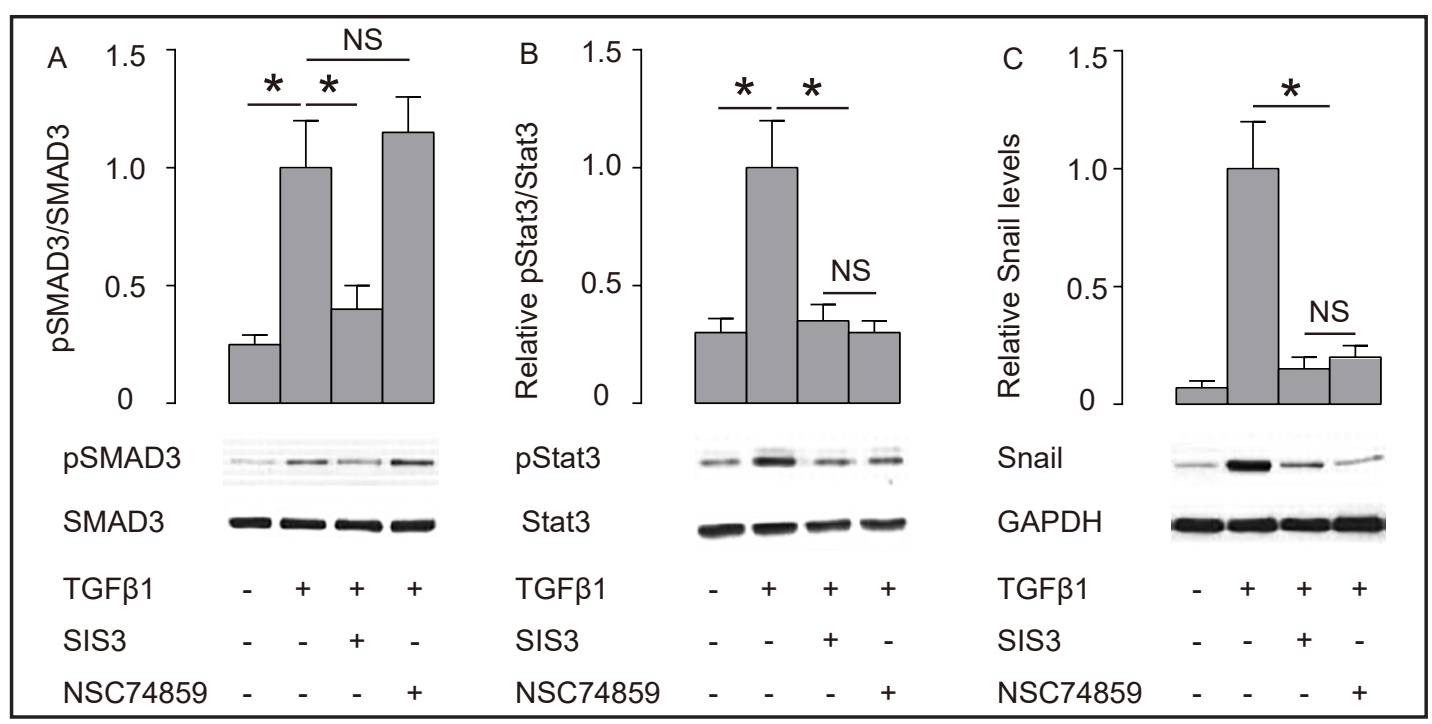

Fig. 3. TGF $\beta 1$ induces EMT of renal TECs through SMAD3/Stat3 signaling Primary mouse TECs were treated with $10 \mathrm{ng} / \mathrm{ml}$ TGF $\beta 1$ and a specific SMAD3 phosphorylation inhibitor (SIS3) at $5 \mu \mathrm{mol} / \mathrm{l}$, or a specific Stat3 phosphorylation inhibitor NSC74859 at $5 \mu \mathrm{mol} / \mathrm{l}$. Cells were analyzed 8 hours after treatment. (A-C) Western blotting for pSMAD3 and SMAD3 (A), for pStat3 and Stat3 (B), and for Snail (C). N=5. *p<0.05. NS: non-significant.

early after TGF $\beta 1$ treatment. Moreover, the expression pattern of mRNA and protein was very similar (Fig. 2D-E). These data suggest that Snail may be activated primarily at the transcription level by TGF $\beta 1$.

\section{TGFB1 induces EMT of renal TECs through SMAD3/Stat3 signaling}

Finally, we examined whether the recent report about activation of Snail in tumors by TGF $\beta / S M A D 3 / S t a t 3$ may be also function in renal TECs [11]. Primary mouse TECs were treated with TGF $\beta 1$ and a specific SMAD3 phosphorylation inhibitor (SIS3), or a specific Stat3 phosphorylation inhibitor NSC74859. Cells were analyzed 8 hours after treatment. We found that TGF $\beta 1$ induced phosphorylation of SMAD3 and Stat3, as well as activation of Snail (Fig. 3A-C). Suppression of SMAD3 phosphorylation by SIS3 prevented both TGF 31 -induced phosphorylation of Stat3 and TGF $\beta 1$-induced activation of Snail, but suppression of Stat3 phosphorylation by NSC74859 prevented only TGF $\beta 1$-induced activation of Snail, without affecting TGF $\beta 1$-induced phosphorylation of SMAD3 (Fig. 3A-C). These data suggest that TGFß1 may trigger EMT of renal TECs through SMAD3/Stat3 signaling.

\section{Discussion}

EMT plays critical roles in embryonic development, tumor initiation and progression and organ remodeling. TGF $\beta 1$ greatly increased in the injured and inflamed kidney, mainly released by myofibroblasts and macrophages. TGF $\beta 1$ has been characterized as a major inducer of EMT in renal TECs [13]. The major downstream target of TGF $\beta$ receptor signaling is SMAD3, but its phosphorylated/activated form did not directly bind to the promoter region of EMT-associated genes. Hence, the factors that directly contribute to transcriptional activation of EMT-associated genes and activated by pSMAD3 need to be determined.

Very recently, Stat 3 was shown as a mediator that synergizes TGF $\beta$ and Ras signals in tumor cells. This specific function of Stat3 was supported by the factors that several Stat3 mutants lacking transcriptional activity failed to enhance Snail transcription but the wildtype Stat3 did, and Stat3 appeared to enhance Snail induction via its dissociation from 
Protein inhibitor of activated STAT3 (PIAS3) by TGF 31 in cooperation with Ras signals [11]. Moreover, activated Stat3 is known to be a potent activator of Snail, through a variety of mechanisms [11, 14-16]. Since we detected upregulation of pStat3 in the injured kidney, we tried to figure out a direct link between SMAD3 signaling and Stat3 activation as well as Snail expression in the current study. In a set of gain-of-function and loss-of-function experiments, we found out that TGF 31 -induced phosphorylation of SMAD3 indeed promotes transcriptional activation of Snail, through Stat3 signaling. The pSMAD3 may bind to PIAS3, resulting in dissociation of PIAS3 from Stat3, allowing Stat3 to be phosphorylated and then target Snail promoter to activate it.

Yadak et al. showed that IL-6 overexpression in neck tumor cells or immortalized oral epithelial cells significantly induced the expression of mesenchymal marker, vimentin, while repressing E-cadherin expression via the JAK/Stat3/Snail signaling pathway [16]. Moreover, Lee et al. showed that Stat3 down-regulates Snail expression levels and hence suppresses EMT of colorectal cancer cells via augmentation of Snail degradation by regulating phosphorylation of glycogen synthase kinase (GSK) $3 \beta$ [15]. Furthermore, Liu et al. reported that Stat3 knockdown effectively suppressed Snail expression and blocked motility and invasion in atypical teratoid/rhabdoid tumor cells [14]. In addition, in this study, the authors used chromatin immunoprecipitation assay to demonstrate that STAT3 directly binds to Snail promoter [14].

\section{Conclusion}

To summarize the findings from all these studies, here we propose a model of crosstalk between SMAD3 signaling and Stat3 signaling that regulate the Snail expression by TGF $\beta 1$. Understanding this molecular signaling may provide basic knowledge to promote invention of novel strategies for kidney functional preservation against renal fibrosis.

\section{Acknowledgements}

The study was supported by National Natural Science Foundation of China (NO: 81400721,81373227 and 81470947) and Natural Science Foundation of Hunan Province (NO: 2016JJ3173 and 2017JJ2002).It was also supported by a research grant (201605) from the Hunan Academy of Chinese Medicine.

\section{Disclosure Statement}

The authors have declared that no competing interests exist.

\section{References}

1 Gou R, Chen J, Sheng S, Wang R, Fang Y, Yang Z, Wang L, Tang L: KIM-1 Mediates High Glucose-Induced Autophagy and Apoptosis in Renal Tubular Epithelial Cells. Cell Physiol Biochem 2016;38:2479-2488.

2 Guan X, Qian Y, Shen Y, Zhang L, Du Y, Dai H, Qian J, Yan Y: Autophagy protects renal tubular cells against ischemia / reperfusion injury in a time-dependent manner. Cell Physiol Biochem 2015;36:285-298.

- 3 Hu J, Zhu Q Li PL, Wang W, Yi F, Li N: Stem cell conditioned culture media attenuated albumin-induced epithelial-mesenchymal transition in renal tubular cells. Cell Physiol Biochem 2015;35:1719-1728.

4 Wu H, Huang T, Ying L, Han C, Li D, Xu Y, Zhang M, Mou S, Dong Z: MiR-155 is Involved in Renal IschemiaReperfusion Injury via Direct Targeting of FoxO3a and Regulating Renal Tubular Cell Pyroptosis. Cell Physiol Biochem 2016;40:1692-1705. 


\section{Cellular Physiology Cell Physiol Biochem 2017;42:2552-2558 \begin{tabular}{l|l} 
DOI: 10.1159/000480216 & Ond Biochemistry Published 2017 The Author(s). Published by S. Karger AG, Basel \\
www.karger.com/cpb
\end{tabular}}

Liu et al.: Stat3 Signaling Regulates RF-Associated EMT of Tubular Ecs

5 Ma W, Tao L, Wang X, Liu Q, Zhang W, Li Q, He C, Xue D, Zhang J, Liu C: Sorafenib Inhibits Renal Fibrosis Induced by Unilateral Ureteral Obstruction via Inhibition of Macrophage Infiltration. Cell Physiol Biochem 2016;39:1837-1849.

6 Zhao J, Wang L, Cao AL, Jiang MQ, Chen X, Wang Y, Wang YM, Wang H, Zhang XM, Peng W: HuangQi Decoction Ameliorates Renal Fibrosis via TGF-beta/Smad Signaling Pathway In vivo and In vitro. Cell Physiol Biochem 2016;38:1761-1774.

7 Kim J, Kong J, Chang H, Kim H, Kim A: EGF induces epithelial-mesenchymal transition through phosphoSmad2/3-Snail signaling pathway in breast cancer cells. Oncotarget 2016;7:85021-85032.

8 Li D, Sun H, Sun WJ, Bao HB, Si SH, Fan JL, Lin P, Cui RJ, Pan YJ, Wen SM, Zheng XL, Yu XG: Role of RbBP5 and H3K4me3 in the vicinity of Snail transcription start site during epithelial-mesenchymal transition in prostate cancer cell. Oncotarget 2016;7:65553-65567.

-9 Yang SW, Zhang ZG, Hao YX, Zhao YL, Qian F, Shi Y, Li PA, Liu CY, Yu PW: HIF-1alpha induces the epithelialmesenchymal transition in gastric cancer stem cells through the Snail pathway. Oncotarget 2017;8:95359545.

10 Massague J, Wotton D: Transcriptional control by the TGF-beta/Smad signaling system. EMBO J 2000;19:1745-1754.

11 Saitoh M, Endo K, Furuya S, Minami M, Fukasawa A, Imamura T, Miyazawa K: STAT3 integrates cooperative Ras and TGF-beta signals that induce Snail expression. Oncogene 2016;35:1049-1057.

12 Wen JG: Partial unilateral ureteral obstruction in rats. Neurourol Urodyn 2002;21:231-250.

13 Chen YS, Mathias RA, Mathivanan S, Kapp EA, Moritz RL, Zhu HJ, Simpson RJ: Proteomics profiling of Madin-Darby canine kidney plasma membranes reveals Wnt-5a involvement during oncogenic H-Ras/TGFbeta-mediated epithelial-mesenchymal transition. Mol Cell Proteomics 2011;10:M110 001131.

14 Liu WH, Chen MT, Wang ML, Lee YY, Chiou GY, Chien CS, Huang PI, Chen YW, Huang MC, Chiou SH, Shih YH, Ma HI: Cisplatin-selected resistance is associated with increased motility and stem-like properties via activation of STAT3/Snail axis in atypical teratoid/rhabdoid tumor cells. Oncotarget 2015;6:1750-1768.

15 Lee J, Kim JC, Lee SE, Quinley C, Kim H, Herdman S, Corr M, Raz E: Signal transducer and activator of transcription 3 (STAT3) protein suppresses adenoma-to-carcinoma transition in Apcmin/+ mice via regulation of Snail-1 (SNAI) protein stability. J Biol Chem 2012;287:18182-18189.

16 Yadav A, Kumar B, Datta J, Teknos TN, Kumar P: IL-6 promotes head and neck tumor metastasis by inducing epithelial-mesenchymal transition via the JAK-STAT3-SNAIL signaling pathway. Mol Cancer Res 2011;9:1658-1667. 